\title{
SONS FRICATIVOS SURDOS
}

\author{
Voiceless fricatives sounds
}

\author{
Carla Aparecida Cielo ${ }^{(1)}$, Maísa Tatiana Casarin (2)
}

\begin{abstract}
RESUMO
Tema: características dos sons fricativos surdos. Objetivo: propor uma revisão da literatura pertinente às características acústicas, fonéticas e fonológicas dos fonemas fricativos surdos que integram o sistema fonológico do Português. Além disso, são descritas suas aplicações na terapia vocal. Conclusões: os fricativos são fonemas agudos, abrangendo de 2500 a $8000 \mathrm{~Hz}$; são plenamente adquiridos até os 3:7 anos de idade; o /s/ que também é o mais afetado em casos de frênulo lingual curto; a omissão do /s/ é uma das ocorrências mais freqüentes na alfabetização; sendo que, no desvio fonológico e na fissura lábio-palatina, freqüentemente ocorre comprometimento de toda a classe de fricativos. Na avaliação de voz, os fricativos são mencionados com as medidas de TMF e relação s/z, bem como seu uso como sons de apoio na fonoterapia.
\end{abstract}

DESCRITORES: Voz; Fonética; Treinamento da Voz; Fonoterapia

\section{INTRODUÇÃO}

Os fonemas fricativos surdos têm importância singular na prática fonoaudiológica, pois são alvo de interesse e estudos tanto nas áreas da audição, linguagem (oral e escrita), motricidade oral, quanto na área de voz ${ }^{1-6}$.

Apesar do grande número de pesquisas realizadas nas áreas de fonologia, ainda são muito poucos os estudos sobre o seu uso na área de voz. Os fonemas fricativos surdos são de fácil produção e podem ser utilizados na avaliação quantitativa e qualitativa da voz, no treinamento do controle respiratório, e no treinamento da dinâmica fonatória. Também podem ser utilizados como sons de apoio na terapia das disfonias e nos pós-operatórios imediatos de laringe por não solicitarem a fonte glótica ${ }^{6-14}$.

As consoantes recebem o nome de fricativas quando o fluxo de ar passa por um grande estreitamento na cavidade oral, suficiente para produzir uma turbulência aérea, como ocorre nos fonemas /f/, / / /, /s/, /z/, / //, /Z/. Convém lembrar que todos os fonemas sonoros apresentam menor intensidade do

(1) Fonoaudióloga; Professora Adjunta do Departamento de Fonoaudiologia da Universidade Federal de Santa Maria em Nível de Graduação e Pós-Graduação; Doutora em Lingüística Aplicada pela Pontifícia Universidade Católica do Rio Grande do Sul.

(2) Fonoaudióloga; Pesquisadora do Centro de Estudos da Linguagem e Fala da Universidade Federal de Santa Maria; Mestre em Distúrbios da Comunicação Humana pela Universidade Federal de Santa Maria. que seus correspondentes surdos e são, também, um pouco mais graves devido ao acoplamento da fonte glótica à fonte friccional ${ }^{1,2}$.

Algumas pesquisas mostram que esses fonemas são completamente adquiridos até os 3:7 anos de idade ${ }^{15,16}$. Na literatura internacional, existem muitas pesquisas voltadas para a caracterização dos fonemas fricativos surdos em populações específicas, como, por exemplo, em casos de pacientes com Mal de Parkinson e afasia, dentre outras desordens que alteram a fala ${ }^{17-23}$.

As publicações que abordam o uso dos fonemas fricativos na área da voz são escassas, apesar de sua importância clínica. A utilização desses fonemas inicia-se na avaliação da voz, com as medidas de tempo máximo de fonação de /s/ e de /z/, e da relação $\mathrm{s} / \mathrm{z}$, e estende-se durante o processo de tratamento das disfonias, quando são utilizados como técnica de terapia ${ }^{6-14}$.

Com base no que foi exposto, o objetivo principal deste artigo é propor uma revisão da literatura pertinente às características acústicas, fonéticas e fonológicas dos fonemas fricativos surdos que integram o sistema fonológico do Português. Além disso, serão descritas suas aplicações na terapia vocal.

\section{MÉTODOS}

Foi realizada revisão da literatura sobre o tema proposto nas bases de dados PUBMED, Bireme, LILACS, MEDLINE, da National Library of 
Medicine, utilizando-se as palavras-chave "treinamento da voz", "fonemas fricativos", "fonética", "voz", e "fala" em diferentes combinações, abrangendo o período de janeiro de 2003 até abril de 2008. Algumas obras referenciadas nos trabalhos encontrados também foram consultadas em sites específicos. Foram incluídas referências de livros-texto, artigos de periódicos nacionais e internacionais considerados como base para a caracterização dos fonemas fricativos e sua aplicação à área da voz.

A distribuição e análise dos resultados foram realizadas por meio da comparação das diferentes idéias e dos diferentes resultados de pesquisas encontradas na literatura consultada, agrupandose os mesmos temas em tópicos, havendo muitas vezes, por necessidade lógica do texto, o aparecimento isolado de algum autor.

A discussão dos resultados buscou extrair de cada tópico os aspectos mais relevantes relacionando-os com suas fontes e apresentando uma avaliação crítica dos mesmos.

\section{REVISÃO DA LITERATURA}

Características acústicas e fonéticas dos fonemas fricativos

Os fonemas fricativos são as consoantes mais fracas e mais agudas do português brasileiro. Os fonemas /s/ e /z/ são fonemas fortes, agudos, com uma faixa de freqüência acima de $4.500 \mathrm{~Hz}$, chegando a $8.000 \mathrm{~Hz}$ no português brasileiro. Os fonemas / $/$ e e /3/ também apresentam faixa de freqüência ampla, semelhante a/s/ e/z/, porém mais grave, entre 2.500 e $6.000 \mathrm{~Hz}^{2}$.

Os fonemas fricativos que compõem o sistema fonológico do português brasileiro distinguem-se quanto aos seguintes pontos de articulação: lábiodentais (/f/, /v/), alveolares (/s/, /z/), e palatais / $/ /$, /3/). Também existem as fricativas velares e glotais que têm sido utilizadas como formas variantes dos $/ r /$ dialetais. Em cada um desses pontos de articulação, ocorrem os pares sonoro e surdo ${ }^{1,2}$.

Os resultados de um estudo que abordou as contribuições da semântica e das informações faciais para o reconhecimento dos fonemas fricativos surdos da língua inglesa, por adultos com audição normal, sugerem que a percepção apurada desses fonemas deriva de uma combinação de informações acústicas, lingüísticas, e visuais ${ }^{21}$.

Em estudo sobre o cruzamento de dados lingüístico-acústicos dos fonemas fricativos surdos em sete línguas, o fonema /s/ foi o que mostrou maior variação inter-falantes ${ }^{22}$.

As informações lingüísticas e visuais, aliadas às informações acústicas, também podem auxiliar a percepção apurada dos fonemas fricativos surdos, uma vez que os fricativos são fonemas com turbulência aérea evidenciada e de altas freqüências, mas também com uma articulação de certa forma visível utilizando-se de lábios e língua e auxiliando o ouvinte com essas pistas visuais. Além disso, a língua falada e o contexto lingüístico também favorecem a identificação de tais fonemas ${ }^{1,2,21}$.

\section{Estudos sobre a aquisição dos fonemas fricativos do português brasileiro}

A aquisição da fonologia do vem sendo bastante estudada nas últimas décadas ${ }^{3}$. Alguns estudos relacionados com os fonemas fricativos merecem destaque pela sua relevância.

Estudo realizado sobre a aquisição das fricativas /f/, / $/ \mathrm{V} /, / \mathrm{J} /, / 3 /$, em crianças com idade entre 1:0 e 3:8 (anos: meses), verificou que o fonema /f/ é adquirido a partir de 1:9; o fonema /v/ $\operatorname{com~1:8;~e~o~}$ fonema $/ \mathrm{J} / \mathrm{com} 2: 10^{3}$. Outra pesquisa que descreveu a aquisição das fricativas /s/ e /z/, por crianças com desenvolvimento fonológico normal e idades entre $1: 0$ a $3: 3$, apontou que o fonema /s/ encontra-se adquirido aos 2:6 na posição de onset, e o fonema / $z /$ encontra-se adquirido aos 2 anos ${ }^{15}$.

No entanto, em outro estudo, realizado com crianças entre 3:0 e 7:0, observou-se o domínio dos fonemas /s/, /f/ e /// aos 3:0, nas posições inicial e final; e o domínio do /3/ aos 3:7, nas posições inicial e final ${ }^{4}$.

Em pesquisa realizada com 72 crianças, com idades entre 1:3 e 2:5, evidenciou-se, como padrão, a emergência da fricativa / / em etapa anterior à da fricativa $/ \mathrm{s} /{ }^{16}$.

\section{Transtornos da articulação}

Estudo americano com três crianças, entre 5:0 e 6:0, que omitiam o fonema /s/ em posição final de palavra, mas preservavam o contraste [+sonoro] desse fonema, produzindo uma duração diferenciada precedendo a vogal, mostrou significativo aumento de duração de vogais tanto em sílabas $\mathrm{C}-\mathrm{V}$, quanto em $\mathrm{C}-\mathrm{V}-\mathrm{C}$, com significativo aumento das vogais que precediam as consoantes sonoras, quando comparadas às surdas ${ }^{17}$.

Os fonemas mais afetados na articulação de 200 pacientes com diagnóstico de Mal de Parkinson idiopático, foram /s/ e /z/ acometidos em $80 \%$ dos casos, e, em menor grau, / $/ \mathrm{/}$ e /3/ em um total de $50 \%$ das ocorrências. Os erros articulatórios ocorreram por haver inadequada elevação da língua e fechamento insuficiente para constrição do fluxo aéreo ${ }^{18}$.

Os fonemas fricativos surdos e sonoros foram estudados em três pacientes com afasia motora, por meio de análise acústica, sugerindo-se que o déficit de produção da fala na afasia anterior não 
ocorre no alto estágio da seleção fonêmica ou planificação, mas na execução articulatória relacionada ao controle laríngeo ${ }^{19}$.

Em pesquisa sobre próteses maxilares implantadas em 30 sujeitos, verificou-se que a porcentagem de sons produzidos corretamente pelos sujeitos que utilizavam prótese total foi significativamente maior do que a dos sujeitos com prótese fixa ${ }^{20}$.

Em estudo conduzido com 13 pacientes falantes do espanhol chileno, avaliou-se o efeito do aparelho ortodôntico lingual fixo na articulação dos sons e a adaptação destes efeitos dentro do primeiro mês de uso do aparelho. Foi realizada avaliação fonoaudiológica em cinco momentos diferentes: antes da instalação do aparelho, imediatamente após a instalação, nas 24 horas posteriores, nos sete dias e um mês após a colocação do aparelho. Observaram-se trocas significativas no ponto de articulação dos fones [d], [s] e [r]. Os fones [d] e [s] mostraram uma resolução favorável dentro do primeiro mês de uso dos aparelhos. No caso da fricativa [s], observou-se que a evolução favorável de sua alteração ocorreu após uma semana da colocação do aparelho, quando a alteração foi corrigida em todos pacientes avaliados ${ }^{23}$.

A pesquisa sobre a aquisição de segmentos consonantais, integrantes da fonologia de diferentes línguas, como exposto anteriormente, tem enfatizado que a emergência das fricativas ocorre em fase subseqüente à emergência de outras classes de consoantes. E, no caso de desvio fonológico, freqüentemente há comprometimento no emprego dos fonemas fricativos ${ }^{4}$.

A estrutura anormal do lábio, do processo alveolar, e do palato em sujeitos portadores de fissura lábio-palatina constituem obstáculos para a correta articulação dos fonemas. A atresia palatal causa distúrbios na pronúncia das consoantes fricativas /s/, /z/, /f/, /v/, /J/, /z/ e nas plosivas /t/, /d/. Ocasionalmente, também é afetada a pronúncia do /f/ e / $/ \mathrm{J}$ que dependem do lábio inferior em contato com os dentes superiores ${ }^{24}$.

Entre os fonemas mais afetados nos indivíduos com fissura lábio-palatina está o fricativo $/ \mathrm{s} /{ }^{25}$.

Em estudo realizado com 54 pacientes que apresentavam ceceio, verificou-se que a tonicidade da sílaba com a fricativa [s] em diferentes contextos silábicos e co-articulação com diferentes vogais não alteraram a incidência do ceceio. As posições iniciais de sílaba, porém, podem ser facilitadoras da produção de [s] e devem, portanto, ser consideradas na escolha do recurso e material a ser utilizado em terapia ${ }^{26}$.

Em estudo sobre a classificação e a interferência do frênulo lingual na fala, observou-se que, dos 1402 sujeitos, $62(9,9 \%)$ apresentavam alterações de fala e, dentre as mais freqüentes, estavam as omissões e substituições do fonema /s/ ${ }^{27}$.

Quanto à aquisição, pode-se afirmar que os fonemas fricativos são plenamente adquiridos até os 3:7 anos de idade ${ }^{3,16}$. Isto significa que a criança os domina antes de entrar para as séries iniciais e iniciar a alfabetização.

Em crianças com desvios fonológicos, freqüentemente há comprometimento do uso dos fonemas fricativos ${ }^{3,16}$. A literatura mostra também o comprometimento da classe inteira de fricativos na fissura lábio-palatina ${ }^{24} \mathrm{e}$ na afasia motora ${ }^{19}$, evidenciando que, além de poderem ser prejudicados em nível cognitivo, como nos desvios fonológicos, os fricativos surdos e sonoros sofrem distúrbios também no nível motor ou de realização como nestes casos. Ainda no nível motor, verificou-se que o /s/ é o fonema mais afetado em casos de frênulo lingual curto ${ }^{27}$, e que, na Doença de Parkinson ${ }^{18}$, a maioria dos fonemas fricativos aparece comprometida em sua articulação, reforçando a importância da classe de fricativos para a comunicação como um todo e a possibilidade de distúrbios em nível mais periférico ou de realização articulatória.

\section{Relações com a ortografia}

Em estudo conduzido para analisar o material escrito de duas crianças da primeira etapa do ensino fundamental com diagnóstico de disortografia, foram analisadas as substituições entre os grafemas "ch" ou "x" e "j" ou "g" para o par de fonemas $/ \mathrm{s} / \mathrm{-} / 3 /$, e os grafemas "f" ou " $\mathrm{v}$ " para o par de fonemas /f/ - /v/. Observou-se uma tendência ligeiramente maior à sonorização, mais saliente em $/ \mathrm{s} /-/ 3 /$ (78\%); depois, /f/ - /v/, com sonorização de 67\%; o único par a apresentar um número maior de dessonorização foi /s/ - /z/, com $61 \%$ das ocorrências ${ }^{5}$.

O fonoaudiólogo pode atuar junto aos professores para o enriquecimento do processo de ensinoaprendizagem da escrita, trabalhando e capacitando os docentes acerca das habilidades em consciência fonológica ${ }^{28}$.

As dificuldades verificadas com relação aos fonemas fricativos, tanto na esfera cognitiva quanto na motora, por sua vez, poderão também ter repercussões na ortografia ${ }^{5,28}$, uma vez que a caracterização mental inapropriada do fonema (nível cognitivo) pode levar à representação gráfica errada, confusa, ou distorcida, na fala e na escrita. Da mesma forma, quando os fonemas são realizados inadequadamente na fala (nível motor), partindose do princípio de que a escrita toma como base a linguagem falada, é possível que a codificação em linguagem escrita ocorra inadequadamente, como mostraram as pesquisas consultadas em relação ao grande número de ocorrências de sonorizações, 
dessonorizações, e omissões de fonemas fricativos na escrita infantil ${ }^{5}$.

\section{Uso clínico no diagnóstico e tratamento das disfonias}

$\mathrm{Na}$ área da voz, pôde-se constatar que as publicações que abordam o uso dos fonemas fricativos surdos são escassas, apesar de sua importância clínica. Conforme a literatura consultada, são três os pontos principais da aplicabilidade dos fricativos na clínica e na pesquisa em voz.

A utilização dos fonemas fricativos inicia-se na avaliação de voz ${ }^{6,8,10,12-14}$, com as medidas dos tempos máximos de fonação (TMF) e relação $s / z$, e estende-se durante o treinamento vocal e o tratamento das disfonias, quando são utilizados como sons de apoio 6,8-12,14.

A investigação dos TMF constitui a parte mais simples da avaliação objetiva quantitativa da voz. Juntamente com a análise acústica, a medida dos tempos máximos de fonação possibilita comparação intra e inter-sujeito, podendo servir como linha de base e de análise tanto da evolução de uma voz profissional como para avaliação do tratamento de um paciente disfônico ${ }^{6,8,10,12-14}$.

O tempo máximo de fonação é a duração máxima que uma pessoa pode sustentar um som em uma expiração prolongada. Supõe-se que ela seja uma medida do controle fonatório e de "apoio" respiratório. Quando a função respiratória estiver comprometida, haverá uma redução na quantidade de ar disponível para apoiar a fonação, um problema no controle do fluxo de ar. Se o problema ocorre em nível laríngeo, a resistência glótica ao fluxo de ar pode ser reduzida por fechamento glótico inadequado ou aumentado devido à obstrução ou hiperadução 6-8,10-14.

Determinados problemas que afetam o controle motor da fonação podem não inibir ou restringir o TMF, porém, podem afetar a qualidade da emissão. A tarefa é projetada para testar os limites do funcionamento e, como tal, pode revelar fraquezas que não se mostram aparentes em níveis mais baixos de funcionamento. No caso de um indivíduo com tremor essencial, um tremor vocal pode tornar-se crescentemente óbvio à medida que a fonação é sustentada, embora possa ter escapado da percepção durante a fala, constituindo-se, desta forma, a medida do TMF também num dado qualitativo da fonação ${ }^{6-8,10,13}$.

A medida dos TMF constitui uma avaliação quantitativa da voz, obtendo medidas respiratória e fonatória. Mensurações dos TMF das fricativas /s/ e /z/ e da relação /s/ e /z/ são importantes para avaliação da dinâmica fonatória e da eficiência glótica.
$\mathrm{O} / \mathrm{s} /$ mede o controle respiratório, o /z/ adiciona o componente glótico à emissão ${ }^{6-14}$.

Para as medidas dos TMF, é considerado o tempo máximo de sustentação, após inspiração profunda, dos fonemas /a:/, /i:/, /u:/ e dos fonemas fricativos /s:/ e /z:/, bem como da contagem de números. Espera-se que os valores sejam os mesmos, ou com pequena variação entre o fonema surdo e o fonema sonoro, uma vez que a dinâmica fonatória normal é capaz de utilizar a suplência de ar pulmonar de maneira eficiente ${ }^{6-8,10,13}$. Para as medida das fricativas /s/ e/z/, assume-se o pressuposto de que, numa fonação sustentada, um indivíduo com dinâmica fonatória normal é capaz de utilizar a suplência de ar pulmonar de modo eficiente, o que não acontece com os pacientes disfônicos ${ }^{7}$.

A proporção $\mathrm{s} / \mathrm{z}$ é uma medida simples destinada a examinar o efeito da patologia sobre a fonação. O procedimento é utilizado para medir a proporção s/z é bastante direto. O paciente é instruído a inspirar profundamente e, então, sustentar um /s/ durante tanto tempo quanto possível. O examinador deve modelar a tarefa, embora não seja necessário sustentar o modelo maximamente. A tarefa deve ser repetida pelo menos duas vezes pelo paciente, com a duração mais longa tomada como o escore. O mesmo procedimento é utilizado para sustentar o /z/. É melhor usar um cronômetro para obter tais medições ${ }^{6-8,10,13}$.

A proporção é obtida dividindo o valor do TMF de /s/ máximo pelo valor do TMF de /z/ máximo. Embora haja alguma variação, valores de relação s/ $z$ aproximados a 1,0 correspondem à normalidade, ou seja, há equilíbrio entre força aerodinâmica e muscular da laringe e são esperados tanto para crianças como para adultos de diferentes idades. Valores maiores ou iguais a 1,2 são significativos de falta de coaptação correta das pregas vocais à fonação, podendo indicar a presença de uma patologia nessa região. Valores abaixo de 0,8 sugerem aumento exagerado da força muscular da laringe. Quanto maior o valor desta proporção, menor o controle laríngeo à passagem de ar expiratório ${ }^{6-10}$.

É consenso entre os autores ${ }^{6-10,12-14,29,30}$ que as medidas de TMF de fricativos possibilitam comparações de resultados intra e inter-sujeitos, uma vez que são objetivas e fornecem dados quantitativos sobre a sustentação glótica do som, no caso dos fricativos sonoros, mais especificamente o/z/, e sobre o controle aerodinâmico, no caso dos fricativos surdos, mais especificamente o/s/, ambos sinalizando objetivamente, por meio da cronometragem de seus TMF, e subjetivamente, por meio da qualidade se sua emissão, possíveis distúrbios nesses níveis.

Outro ponto consensual na literatura ${ }^{6-14}$ sobre voz refere-se à relação ou proporção entre os TMF 
de /s/ e de /z/, uma vez que a mesma, também de forma objetiva, propõe-se a evidenciar as alterações em nível de sustentação do ar durante a fonação em relação com a sustentação glótica do som, reforçando os achados que se obtêm com os TMF isolados de /s/ e de /z/.

Com relação às médias do TMF, são considerados valores em torno de 20 s como padrão normativo para homens adultos, 14s para mulheres adultas, considerando-se TMF abaixo de 10s significativamente não normais, em um estudo realizado com falantes brasileiros na cidade de São Paulo ${ }^{9}$.

Quanto às medidas da dinâmica respiratória em crianças de quatro a dez anos, constatou-se que os valores de TMF das consoantes /s/ e /z/ apresentaram resultados próximos aos das vogais, mas com decréscimo de no máximo $1,5 \mathrm{~s}$, sendo os valores de /z/ maiores do que os de /s/. Os valores dos TMF das fricativas variaram bastante conforme a idade das crianças, com médias de $8,67 \mathrm{~s}$ para $/ \mathrm{s} / \mathrm{e}$ 10,43 s para $/ \mathrm{z} /$, aos dez anos de idade ${ }^{29}$.

Em estudo sobre os coeficientes de associação e correlação entre os TMF, posição e ângulo de afastamento das pregas vocais, em 86 indivíduos com paralisia unilateral de prega vocal, observouse que a associação e correlação têm significância estatística somente para /z/ com a prega vocal na posição mediana. Os valores de relação s/z encontraram-se superiores a 1,3 com as pregas vocais nas posições para-mediana, intermediária e de abdução, traduzindo incompetência glótica ${ }^{30}$, com provável escape aéreo à fonação.

O treinamento vocal que utiliza uma série de sons facilitadores ou sons de apoio pode ser realizado com os sons fricativos ${ }^{6,11-14}$. O procedimento básico envolve a emissão dos sons fricativos surdos /f/, /s/, e / / contínuos (ou seus correspondentes sonoros /v/, /z/, e /z/) ou em passagem de sonoridade, de um som surdo para sonoro. Os efeitos esperados na utilização desses sons facilitadores são: direção de fluxo aéreo para o ambiente, suavização do ataque vocal, aumento dos TMF sem esforço, coordenação pneumofonoarticulatória. Os autores enfatizam que a aplicação principal é no pós-operatório de lesões de massa e na coordenação pneumofonoarticulatória ${ }^{8-10,12}$.

Em estudo de uma mulher adulta, após a utilização do fricativo sonoro /3/, observou-se que houve redução do ruído glótico, com melhora da relação harmônico-ruído, e diminuição do quociente de contato entre as pregas vocais. A técnica também promoveu a normotensão muscular, o que favorece, juntamente com a maior mobilização da mucosa, a diminuição do contato entre as pregas vocais, gerando menor esforço fonatório e maior projeção vocal ${ }^{11}$.
As técnicas com emissão dos fricativos surdos também são indicados para auxiliar a coordenação pneumofonoarticulatória ${ }^{9-10,12}$.

Os fricativos surdos também são indicados para os pós-operatórios imediatos de laringe, com o objetivo de não prejudicar o processo cicatricial das pregas vocais, e também para implementar o controle de aumento de intensidade, sem causar danos às pregas vocais, uma vez que, para sua produção, a fonte glótica não é solicitada. Para o controle expiratório (fluxo de ar dosado) e compreensão do processo de fonação, a seguinte seqüência pode ser adotada, segundo a literatura: emissão de vogais áfonas longas e sem tensão glótica, sons fricativos surdos e sonoros ${ }^{6,9,10,12-14}$.

Após a inspiração costodiafragmáticoabdominal, o indivíduo produz cada emissão suavemente e com controle voluntário da saída do ar por meio da sustentação intercostal, seguida de sua descontração muscular progressiva e, finalmente, da contração progressiva da musculatura da cinta abdominal até o final do ar de reserva expiratória. Ao expelir o ar emitindo a fricativa / $/$ / com intensidade e força na contração da cinta abdominal (para dentro), prepara-se a musculatura para sua contração durante emissões em fortes intensidades, sem sobrecarga da musculatura laríngea. No entanto, tal exercício não deve ser realizado por pessoas cujo esforço físico pode ser prejudicial à saúde ${ }^{6,10,12,14}$.

As técnicas com emissão dos fonemas fricativos surdos auxiliam a coordenação pneumofonoarticulatória, o direcionamento do fluxo de ar, a dissociação entre aumento de intensidade e esforço vocal, e são indicadas para pós-operatórios laríngeos. As técnicas que utilizam fonemas fricativos surdos, seguidos dos sonoros na mesma emissão, promovem a redução do ataque vocal brusco, a suavização do início da sonorização, proporcionando a harmonia da fonação ao nível da produção do som laríngeo ${ }^{6,9,11-13}$.

O terceiro ponto de convergência da literatura consultada na área de voz ${ }^{6,9,10,12-14}$ evidencia os fonemas fricativos em uso como técnica terapêutica, sendo que os surdos são indicados para os pós-operatórios imediatos de laringe, para o direcionamento do fluxo aéreo expiratório, para a coordenação pneumofonoarticulatória, e para a implementação do aumento de intensidade vocal, sem sobrecarga da laringe, uma vez que não utilizam a fonte glótica. Já os fonemas fricativos sonoros, atuam na musculatura e promovem a mobilização da mucosa das pregas vocais, gerando menor esforço fonatório e maior projeção vocal.

Acredita-se que novas pesquisas na área de técnicas de fonoterapia vocal envolvendo os fonemas fricativos surdos e sonoros deverão ser reali- 
zadas. Esse conhecimento, por sua vez, auxiliará o fonoaudiólogo no processo de indicação dos fonemas fricativos no tratamento das disfonias, com maior especificidade quanto aos seus objetivos de terapia.

\section{COMENTÁRIOS FINAIS}

Neste trabalho, procurou-se descrever, por meio de ampla revisão bibliográfica, as pesquisas mais relevantes e atuais realizadas na área da fonoaudiologia envolvendo os fonemas fricativos surdos. Destacou-se a sua importância nos estudos relacio- nados com a acústica, a fonética, a fonologia, suas relações com a ortografia e a voz.

De modo geral, os fricativos são fonemas agudos, abrangendo de 2500 a $8000 \mathrm{~Hz}$; são plenamente adquiridos até os 3:7 anos de idade; os fricativos surdos são mais variáveis intra e inter-falantes, principalmente o/s/ que muitas vezes encontra-se alterado nos casos de freio lingual curto, na Doença de Parkinson, na fissura lábio-palatina, nos desvios fonológicos e na aquisição da escrita. Na avaliação de voz, os fricativos são mencionados com as medidas de TMF e relação $s / z$, bem como seu uso como sons de apoio na fonoterapia.

\begin{abstract}
Background: characteristics of voiceless fricative sounds Purpose: to review the literature related to acoustic, phonetics and phonological characteristics of voiceless fricative sounds that are part of the phonological system of the Portuguese Language. Furthermore, it describes the use of these sounds in voice therapy. Conclusions: fricatives are acute phonemes comprised between 2500 and $8000 \mathrm{~Hz}$; they are fully acquired up to the age of 3:7 years; the /s/, which is the most affected in cases of short lingual frenum; omission of the $/ \mathrm{s} /$ is one of the most frequent occurrences during literacy education; and in the phonological deviation and labial-palatine fissure the entire class of fricatives is frequently affected. In voice assessment, fricative sounds are mentioned as TMF measurements and $\mathrm{s} / \mathrm{z}$ relationship, and their use as support sounds in speech therapy.
\end{abstract}

KEYWORDS: Voice; Phonetics; Voice Training; Speech Therapy

\section{REFERÊNCIAS}

1. LamprechtRR. Organizador. AquisiçãoFonológica do português: perfil de desenvolvimento e subsídios para terapia. Porto Alegre: Artmed; 2004. 232p.

2. Russo I, Behlau M. As pistas acústicas das vogais e consoantes. In: Russo I, Behlau M. Percepção da fala: análise acústica do português brasileiro. São Paulo: Lovise; 1991. p.25-50.

3. Oliveira CC. Perfil das fricativas /f/, /v/, /Š/, /Ž/ do português brasileiro. Letras de Hoje. 2003; 38(2):97-110.

4. Wertzner H. Aquisição da articulação: um estudo em crianças dos três aos sete anos. Est Psicol. 1994; 11(1/2):11-21.

5. Mousinho R. O traço de sonoridade em crianças disortográficas. Rev Fono Atual. 2003; 24:28-46.

6. Colton R, Casper J. Compreendendo os problemas da voz: uma perspectiva fisiológica ao diagnóstico e ao tratamento. Porto Alegre: Artes Médicas; 1996. 386p.

7. Rossi DC, Munhoz DF, Nogueira CR, Oliveira CM, Britto ATBO. Relação do pico de fluxo expiratório com o tempo de fonação em pacientes asmáticos. Rev CEFAC. 2006; 8(4):509-17.

8. Behlau M, Rodrigues S, Azevedo R, Gonçalves MI, Pontes P. Avaliação e terapia da voz. In: Lopes Filho OC. Tratado de fonoaudiologia. São Paulo: Roca; 1997. p.607-58.

9. Behlau M, Pontes P. Abordagem global na reabilitação vocal. In: Behlau M, Pontes P. Avaliação e tratamento das disfonias. São Paulo: Lovise; 1995. p. $189-262$.

10. Pinho SMR. Avaliação e tratamento da voz. In: Fundamentos em fonoaudiologia: tratando os distúrbios da voz. Rio de Janeiro: Guanabara Koogan; 1998. p. 1-37.

11. Cielo CA, Siqueira MA, D’Ávila $H$. Efeitos da técnica fonoterapêutica de fricativo sonoro /3/ na voz: análise de um caso. Rev Soc Bras Fonoaudiol. 2005; 10(4):232-5.

12. Boone DR, McFarlane SC. A voz e a terapia vocal. Porto Alegre: Artes Médicas; 1994.

13. Behlau M, Madazio G, Feijó D, Pontes P. Avaliação de voz. In: Behlau M. Voz: o livro do especialista. Rio de Janeiro: Revinter; 2001. p.85-180. 
14. Pinho SMR, Tsujji DH. Avaliação funcional da laringe em cantores. Acta Awho. 1996; 15(2):87-93.

15. Sávio CB. Aquisição das fricativas /s/ e /z/ do português brasileiro. Letras de Hoje. 2001; 36(125):721-7.

16. Matzenauer CLB. A aquisição das fricativas coronais com base em restrições. Letras de Hoje. 2003; 38:123-35.

17. Plonsker LS, Petrosino L, Colcord RD. Differential vowel duration associated with children's wordfinal fricative deletion. Percept Mot Skills. 2004; 93(1):31-6.

18. Torres OC, Leon MP, Alvarez EG, Alvarez LG, Maragoto CR, Rivera OR. Particularidades articulares de la disartria Parkinsoniana. Rev Mexicana de Neurociência [periódico online] 2001; 2(4). Disponível em URL: http://imbiomed.com/1/1/ articulos.php?id_revista=91\&id_ejemplar=1425/.

19. Kurowski K, Hazen E, Blumstein SE. The nature of speech production impairments in anterior aphasics: an acoustic analysis of voicing in fricative consonants. Brain Lang. 2003; 84(3):353-71.

20. Heydecke G, Mcfarland DH, Feine JS, Lund JP. Speech with maxillary implant prostheses: ratings of articulation. J Dent Res. 2004; 83(3):236-40.

21. Jongman $A$, Wang $Y$, Kim BH. Contributions of semantic and facial information to perception of nonsibilant fricatives. J Speech Lang Hear Res. 2003; 46(6):1367-77.

22. Gordon M, Barthmaier P, Sands K. A crosslinguistic acoustic study of voiceless fricatives. J Int Phon Ass. 2002; 32:141-74.
23. Villanueva $P$, Lizana $M L$, Huber $H$, Morán $D$, Fernández MA, Palomino HM. Modificaciones en la articulación de fones en pacientes con aparato ortodóntico fijo lingual. Rev CEFAC. 2007; 9(4):483-89.

24. Leite ICG, Magina CM, Maciel CTV, Schettino CS, Condé EM, Soares CF. Fissuras labiopalatinas: considerações relevantes para o fonoaudiólogo. J Bras Fonoaudiol. 2001; 1(6):66-70.

25. Matos HM. La articulación del habla em indivíduos com hendiduras labiopalatinas corregidas: estúdio de dos casos. Rev CEFAC. 2006; 8(2):186-97.

26. Leite AF, Silva SB, Britto ATBO, Ninno CQMSD. Caracterização do ceceio em pacientes de um centro clínico de fonoaudiologia. Rev Soc Bras Fonoaudiol. 2008; 13(1):30-6.

27. Marquesan IQ. Frênulo de lingual: classificação e interferência na fala. Rev CEFAC. 2003; 5(4):341-5.

28. Moura SRS, Cielo CA, Nogaro A. Crianças bilíngües alemão/português: dessonorizações na escrita em português. Rev Fono Atual. 2005; 34:28-46.

29. Fabron EMG, Santos GR, Omote S, Perdoná GC. Medidas da dinâmica respiratória em crianças de quarto a dez anos. Pró-Fono. 2006; 18(3):313-22.

30. Steffen LM, Moschetti MB, Steffen N, Hanayama EM. Paralisia unilateral de prega vocal: associação e correlação entre tempos máximos de fonação, posição e ângulo de afastamento. Rev Bras Otorrinolaringol. 2004; 70(4):450-5.

RECEBIDO EM: 15/07/2007

ACEITO EM: 26/05/2008

Endereço para correspondência:

Rua Coronel Niederauer, 917/407

Santa Maria - RS

CEP: 97015-121

Tel/Fax: (55) 3286-4963

E-mail: fonoaud@terra.com.br 